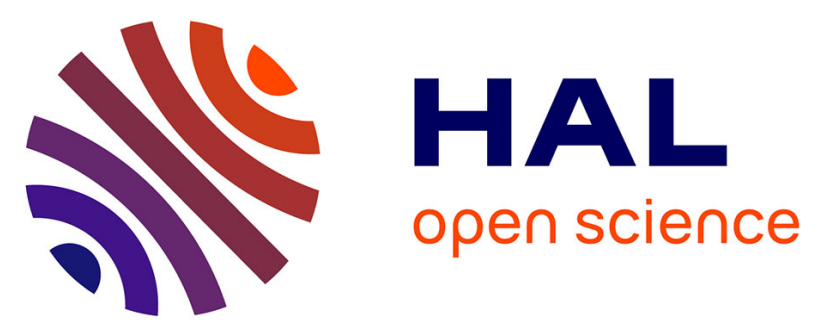

\title{
L'impact des distances psychologiques et des valeurs de distances de pouvoir sur le jugement socio-moral vis-à-vis de transgressions et de conduites atypiques en milieu professionnel
}

\author{
Laurent Auzoult, Sid Abdellaoui, Bernard Gangloff
}

\section{To cite this version:}

Laurent Auzoult, Sid Abdellaoui, Bernard Gangloff. L'impact des distances psychologiques et des valeurs de distances de pouvoir sur le jugement socio-moral vis-à-vis de transgressions et de conduites atypiques en milieu professionnel. Psychologie du travail et des organisations, 2015, 21 (4), pp.380-394. 10.1016/S1420-2530(16)30005-X . hal-01052634

HAL Id: hal-01052634

https://hal-univ-paris8.archives-ouvertes.fr/hal-01052634

Submitted on 7 Feb 2018

HAL is a multi-disciplinary open access archive for the deposit and dissemination of scientific research documents, whether they are published or not. The documents may come from teaching and research institutions in France or abroad, or from public or private research centers.
L'archive ouverte pluridisciplinaire HAL, est destinée au dépôt et à la diffusion de documents scientifiques de niveau recherche, publiés ou non, émanant des établissements d'enseignement et de recherche français ou étrangers, des laboratoires publics ou privés. 


\title{
L'impact des distances psychologiques et des valeurs de distances de pouvoir sur le jugement socio-moral vis-à-vis de transgressions et de conduites atypiques en milieu professionnel
}

\section{The impact of psychological distance and power distance values on the socio- moral judgment vis-à -vis transgressions and atypical behaviors in the workplace}

\author{
Laurent Auzoult* Sid Abdellaoui** \& Bernard Gangloff*** \\ * Université de Franche Comté, Laboratoire de Psychologie (EA 3188), 30-32 rue \\ Mégevand, 25030 Besançon Cedex. laurent.auzoult@univ-fcomte.fr \\ ** Université de Rouen, 1 rue Th. Beckett, 76821, Mont Saint-Aignan, Laboratoire de \\ Psychologie Sociale de Paris 8 (EA 4386). siduniversite@ yahoo.fr \\ *** Université de Rouen, 1 rue Th. Beckett, 76821, Mont Saint-Aignan, Laboratoire de \\ Psychologie Sociale de Paris 10 (EA 3984). bernard.gangloff@univ-rouen.fr
}

Résumé :

On étudie auprès de 178 sujets la relation entre les valeurs de distances de pouvoir, les distances psychologiques et les jugements de sévérité et de rejet de conduites transgressives et/ou atypiques. Les résultats révèlent que le champ des conduites au travail se structure autour des valeurs et des distances psychologiques, l'accentuation des distances conduisant à augmenter la sévérité vis-à-vis des déviances. Ces résultats suggèrent qu'il est nécessaire de considérer les jugements vis-à-vis des conduites déviantes au travail du point de vue des différents registres normatifs invoqués par les salariés.

\section{Abstract :}

We studied the relationship between the value of power distance, the psychological distances and sociomoral judgments of severity and rejection. The results reveal that judgments are structured by values and psychological distances. When the distances increase the judgements are more severe. These results suggest that it is necessary to consider the judgments on deviant behavior at work in relation to normative criteria used by workers.

Mots clés: Distance de pouvoir, distance psychologique, jugement sociomoral

Key-words: Power distance, psychological distance, sociomoral judgment 


\section{Introduction}

Quelle que soit la forme qu'elle prend, économique, sexuelle, physique, psychologique, etc., et la façon de la considérer (abus, exclusion, déviance, harcèlement, agression, incivilités, etc.), la violence au travail pose dès son appréhension la question de son acceptabilité puis de son traitement. En effet, on sait que la modification des conduites passe par une phase initiale de prise de conscience de la gravité des conduites déviantes. Or, la prise en compte de la violence apparaît d'emblée comme un phénomène empreint de subjectivité résultant d'un construit social (Jauvin, 2003). En effet, on observe une large variabilité au niveau de la façon dont tout un chacun perçoit puis se conduit visà-vis de la violence qu'il rencontre au travail. Cette variabilité pourrait traduire des différences quant au registre évaluatif qui sert de référence pour évaluer telle ou telle violence professionnelle. Dans ce cas, il serait possible de considérer cette question sous l'angle du jugement socio-moral. L'étude que nous présentons s'inscrit dans cette perspective.

\subsection{Le jugement socio-moral}

Dans la manière de considérer le fonctionnement du jugement moral, on s'est longtemps appuyé sur une approche développementale pour aboutir à un questionnement davantage psychosocial (Shweder, 1982 ; Emler, 1987 ; Tostain, 1999 a \& b). Initialement, en référence à Piaget (1932), Kohlberg $(1958$; 1981) décrit le développement du jugement moral-cognitif à partir d'une séquence de six stades répartis en trois niveaux : pré-conventionnel, conventionnel et post-conventionnel. Dans cette perspective le développement de la moralité procéderait successivement d'un dépassement des obligations et de l'égocentrisme, puis des règles et de la conventionnalité, pour donner lieu à une gestion cognitive de grands principes universels (Blasi, 1988 ; VandenplasHolper, 1999).

D'autres facteurs que ceux relatifs aux développements psychocognitifs sont déterminants quant à l'intériorisation et l'expression des valeurs sociomorales. Buss (1979) indique que les principes moraux doivent tenir compte de l'ensemble des sources de variations et notamment les conditions sociales continuellement changeantes. De fait, la perception qu'a une personne des bonnes ou mauvaises conduites ainsi que celle de leurs conséquences dépend de son degré de socialisation, de ses propres références identitaires et des enjeux de la situation (Bandura, 1986 ; Tap, 1988 ; Fischer, 1990 ; Roché, 2001 ; Abdellaoui, 2000 ; Blatier, 1998). Ce point de vue s'exprime également à travers la conception de Nucci et Turiel (Nucci \& Turiel, 1978 ; Nucci, 1981; Turiel, 1983). Selon eux, le jugement sociomoral s'organise autour de trois dimensions que sont la moralité qui relève d'impératifs universels partagés quel que soit le contexte, la convention liée aux appartenances sociales et aux 
contextes culturels et le registre personnel qui est lié aux choix privés de la personne. Au delà de son aspect topologique, le modèle de Turiel insiste sur l'importance des interactions sociales directes ou symboliques sur l'élaboration de ces registres de jugement. Globalement, les évolutions théoriques dans ce domaine conduisent à attribuer une importance de plus en plus grande au contexte (Bègue, 1998).

Notamment, l'emprise du contexte sur les jugements socio-moraux est illustrée dans une série de travaux récents (Eyal, Liberman, Trope, 2008) qui mettent en avant la notion de distance psychologique pour rendre compte des variations de perceptions de conduites. Ces études révèlent, notamment, qu'une personne aura tendance à nuancer sa référence aux principes moraux par l'usage du contexte dans lequel s'effectue l'acte en fonction de la distance psychologique qu'il entretient vis-à-vis de l'acte qu'il juge, la réduction de la distance conduisant à une focalisation sur le contexte. Dans ce cadre, les actes sont d'autant plus distants qu'ils se produisent dans un passé ou un futur lointain du sujet, qu'ils se produisent en un endroit d'autant plus éloigné du sujet, qu'ils sont hypothétiques où vécus par autrui plutôt que par le sujet lui même. Précisément, ce processus amène les personnes à juger plus gravement des actes de transgression et de manière plus vertueuse les actes moraux lorsqu'elles sont psychologiquement distantes de l'acte plutôt que proches de celui-ci.

\subsection{Problématique}

La problématique de la violence au travail est le plus souvent abordée sous l'angle des comportements antisociaux au travail (CAAT). Ces derniers renvoient aux comportements déviants vis-à-vis des normes admises dans l'organisation, que ces comportements visent le fonctionnement organisationnel (production, propriété) ou ceux qui y évoluent (violence physique et psychologique). Ce type de typologie ne prend pas en compte les distinctions qui pourraient être produites spontanément entre les conduites transgressant des normes relatives (registre conventionnel du modèle de Turiel) ou des normes universelles (registre moral) voire simplement des conduites atypiques qui ne relèvent pas de transgression mais peuvent faire l'objet d'un jugement de sévérité et de conduites répressives de la part de celui ou celle qui les juge.

Dans cette étude, nous envisagerons des conduites qui peuvent apparaître problématiques pour autrui ou pour l'organisation soit parce qu'elles apparaissent violentes (menaces, vol, dégradation, infractions) soit parce qu'elles apparaissent déviantes par leur singularité, cet ensemble de conduites étant susceptible de faire l'objet d'un contrôle social dans le cadre d'une organisation. En tant que tel, nous envisagerons les jugements portés vis-à-vis de ces conduites comme relevant des conduites de pouvoir. On sait, 
effectivement, que le rapport à l'autorité détermine la façon dont s'élaborent les jugements socio-moraux (Masson-Maret \& Steiner, 2004 ; Mulder, Verboon \& De Cremer, 2009) et que de manière générale les conduites d'évaluation sont étroitement liées à l'affirmation et à la préservation du pouvoir (Gangloff, $1995 ; 1996$; $1997 ; 2000 ; 2002)$.

D'un point de vue explicatif, nous ferons appel au contexte, à travers la notion de distance psychologique, pour rendre compte de la relation vis-à-vis des conduites problématiques, transgressives ou atypiques. Précisément, nous reprendrons l'hypothèse selon laquelle plus la distance vis-à-vis de la conduite problématique est importante et plus les jugements deviennent sévères vis-à-vis d'elle. Notamment, nous ferons l'hypothèse que les personnes jugent d'autant plus sévèrement les conduites transgressives ou atypiques qu'ils sont susceptibles de les avoir commises eux-mêmes plutôt que lorsqu'ils pensent ne pas les avoir commises (hypothèse 1). A titre exploratoire, nous étendrons nos investigations à la perception des distances vis-à-vis de l'objet quand cette dernière concerne autrui, les asymétries entre soi et autrui ayant été mises en évidence dans le rapport aux normes (Codol, 1975) et plus généralement au niveau des jugements socio-moraux (Abdellaoui, Auzoult \& Personnaz, 2010).

De façon à resituer l'étude des distances psychologiques dans la problématique du pouvoir, nous étendrons cette hypothèse aux distances de pouvoir, à travers la notion de valeur de distances de pouvoir (Hofstede, 1989 ; 2001) qui traduit le degré d'inégalité entre les individus que la population considère comme normal (Caramelli, 2006 ; Eylon \& Au, 1999). Ce type de valeur traduisant une distance sociale de pouvoir est susceptible d'expliquer comment s'élaborent des jugements de sévérité et des conduites coercitives, ces valeurs étant liées, entre autres, aux pratiques managériales (House, Javidan, Hanges \& Dorfman, 2002 ; Rom \& HI Hsu, 2002 ; Tan \& Chon, 2003 ; Van de Vliert \& Smith, 2004 ; Carson, 2005 ; Kull \& Wacker, 2009) mais aussi aux attentes d'inégalités en matière de gestion des personnes (Brockner, Ackerman, Greenberg, Gelfand, Francesco, Chen, Leung, Bierbrauer, Gomez, Kirkman \& Shapiro, 2001) ou de perception de justice organisationnelle (Lee, Pillutla \& Law, 2000). Globalement, les études dans ce domaine traduisent la propension des salariés adhérant à de fortes distances de pouvoir à attendre et valoriser les inégalités sociales et professionnelles, notamment au niveau des salaires. Ce faisant, on peut penser que plus les salariés expriment des valeurs de distances de pouvoir fortes et plus ils établissent des distances psychologiques élevées vis-à-vis des autres salariés (hypothèse 2). Le corollaire de cette hypothèse sera que les salariés jugent d'autant plus sévèrement les conduites transgressives ou atypiques qu'ils adhèrent à des valeurs de distances de pouvoir fortes (hypothèse 3). 


\section{Méthodologie}

\subsection{Participants et déroulement}

Cent soixante dix-huit sujets ( 87 hommes et 91 femmes) âgés en moyenne de 37 ans ont participé à l'étude. Il s'agissait de personnes actives, en recherche d'emploi $(\mathrm{N}=18)$, en formation $(\mathrm{N}=14)$, cadres $(\mathrm{N}=30)$, ouvriers $(\mathrm{N}=37)$, employés $(\mathrm{N}=58)$, chefs d'entreprise et professions libérales $(\mathrm{N}=7)$ ou de retraités $(\mathrm{N}=14)$ aussi bien du secteur public que du privé. Ils étaient contactés hors de leur temps de travail et sollicités pour participer à une étude présentée comme portant sur l'évaluation en milieu de travail.

On leur présentait une feuille recto-verso sur laquelle ils pouvaient prendre connaissance dans un premier temps de 27 items décrivant des conduites relevant des trois registres de valeur; morale, conventionnelle et personnelle. Les sujets devaient les évaluer sur deux dimensions (gravité et rejet de l'auteur de l'acte). Puis, ils répondaient à une série de questions permettant d'appréhender leur valeur de distances de pouvoir et les distances psychologiques vis-à-vis des conduites transgressives ou atypiques.

\subsection{Le jugement socio-moral}

Dans un premier temps, nous avons demandé à 105 sujets (30 hommes, 75 femmes, âge moyen $=30$ ans) évoluant en milieu professionnel, de qualifier selon les dimensions de Turiel une série de 50 conduites a priori transgressives et/ou atypiques. Les sujets avaient pour consigne d'indiquer, après avoir pris connaissance d'une scène décrivant une personne effectuant une conduite et une seule dans un milieu professionnel, de préciser à quelle(s) valeur(s) chacune des conduites faisait référence. Pour ce faire, ils avaient à leur disposition, pendant la lecture des 50 items, les trois définitions des valeurs morale (renvoyant à des conduites menaçant l'intégrité psychologique ou physique d'une ou plusieurs personnes), conventionnelle (renvoyant à des conduites ne tenant pas compte des règles de vie sociale) ou personnelle (correspondant à des conduites atypiques n'impliquant pas autrui).

Les résultats de ce pré-test nous ont permis de retenir 27 conduites identifiées comme étant les plus en rapport avec les trois registres de valeur (voir annexe 1). Les items ont été assignés à chaque registre à partir des taux de consensus observés, les 27 items conservés ayant un taux significativement plus important que celui relevant du hasard (Traitement par le $\mathrm{X}^{2}$ d'ajustement $\left(\mathrm{X}^{2}{ }_{\text {observe }}>\mathrm{X}^{2}(\mathrm{p}<.05)=5.99\right.$ avec un $\%$ théorique de $33.3 \%$ pour chaque catégorie de valeur). Les items n'ayant pas été référencés de manière non ambiguë à l'un des trois registres de valeurs ont été éliminés (par ex. Il arrive souvent à Virginie de quitter l'usine avant l'heure en laissant à un de ses 
collègues le soin de badger à la pointeuse à sa place, Il arrive quelques fois à Martine qui est vendeuse en boulangerie de grignoter quelques gâteaux pendant son service, Vincent est directeur des ressources humaines. Il aime bien faire des "allusions » à sa secrétaire et lui passer la main sur les épaules malgré ses protestations). Les taux moyens de consensus inter-sujets sont respectivement de $50 \%$ pour les conduites référant aux valeurs morales, de 51 $\%$ pour celles relevant des valeurs conventionnelles et de $68 \%$ pour celles relevant des valeurs personnelles.

Dans cette étude, les 178 sujets pouvant évaluer la gravité et le rejet des 27 conduites à l'aide d'échelles en 5 points, on observe un alpha de Cronbach de .91 sur l'ensemble des scores de gravité, de .71 pour les scores de gravité portant sur les conduites transgressives de valeur morale, de .66 pour les scores de gravité portant sur les conduites transgressives de valeur conventionnelle et de .70 pour les scores de gravité portant sur les conduites atypiques. De même, on observe un alpha de Cronbach de .88 sur l'ensemble des scores de rejet, de .78 pour les scores de rejet portant sur les conduites transgressives de valeur morale, de .79 pour les scores de rejet portant sur les conduites transgressives de valeur conventionnelle et de .81 pour les scores de rejet portant sur les conduites atypiques.

\subsection{Les distances psychologiques}

La distance psychologique était appréhendée à travers trois questions auxquelles les sujets devaient répondre par oui ou par non. Précisément, on demandait aux sujets s'ils avaient déjà commis certains comportements dont ils avaient évalué la gravité, si des personnes qui leur étaient proches (famille, amis) avaient déjà commis certains de ces comportements ou si un ou plusieurs de leurs collègues avaient déjà commis certains de ces comportements.

\subsection{Les distances de pouvoir}

A partir de divers questionnaires mesurant les valeurs de distances de pouvoir (Hofstede, 2001 ; Earley \& Erez, 1997; House, Javidan, Hanges \& Dorfman, 2002), nous avons élaboré une échelle comportant 9 idées générales renvoyant aux relations de pouvoir dans le monde professionnel (Vous trouvez normal que le pouvoir soit partagé de manière inégale dans les organisations, Les salariés sont supposés obéir à leur supérieur hiérarchique sans poser de question, Les salariés ne devraient pas exprimer leur mécontentement vis-à-vis de leur supérieur hiérarchique, Il est normal que certaines personnes puissent avoir certains privilèges ou symboles de pouvoir (voiture de fonction, par exemple) alors que d'autres n'en ont aucun, Les salariés qui remettent souvent en question l'autorité empêchent parfois leurs directeurs d'être efficaces, Quand un responsable prend une décision les personnels de l'entreprise ne 
doivent pas la remettre en cause, Les managers qui laissent leurs subordonnés participer aux décisions perdent de leur pouvoir, Le règlement d'une entreprise ne doit pas être contesté, même si les salariés estiment que c'est dans l'intérêt de l'entreprise, Il est acceptable qu'il y ait de fortes inégalités de salaire dans les entreprises). Il était indiqué que les idées reflétaient des valeurs générales liées à la culture nationale et aux expériences des sujets et qu'ils devaient exprimer leur propre point de vue sans se soucier de ce que les autres pourraient répondre. Les sujets devaient indiquer leur degré d'accord vis-à-vis de ces 9 items sur des échelles en cinq points, leurs réponses étant moyennées pour donner un score de valeur de pouvoir (L'alpha de Cronbach est de .75 pour les 9 items).

\section{Résultats}

3.1 Les relations entre les distances psychologiques et les jugements socio-moraux

Les sujets attribuent la même gravité aux transgressions de valeurs morales qu'ils estiment $(\mathrm{M}=4.1)$ ou non $(\mathrm{M}=4.2)$ avoir commis des conduites transgressives et/ou atypiques $(\mathrm{F}(1,176)=1.91 ;$ n.s. $)$. Dans le même temps, ils rejettent davantage leurs auteurs lorsqu'ils estiment ne pas avoir commis euxmêmes de conduites transgressives et/ou atypiques $(\mathrm{M}=4.07)$ plutôt que lorsqu'ils estiment en avoir commis $(\mathrm{M}=3.7 ; \mathrm{F}(1,176)=7.51 ; \mathrm{p}<.01)$. De même, les sujets jugent plus gravement $(3.14 / 2.9 ; \mathrm{F}(1,176)=5.64 ; \mathrm{p}<.01)$ et rejettent davantage $(3.02 / 2.72 ; \mathrm{F}(1,176)=4.88 ; \mathrm{p}<.03)$ les auteurs de transgressions de valeurs conventionnelles lorsqu'ils estiment ne pas avoir euxmêmes commis de transgressions ou de conduites atypiques plutôt que lorsqu'ils déclarent en avoir commis. Sur le registre des valeurs personnelles on n'observe pas de différence : les sujets jugent la gravité $(2.31 / 2.26 ; \mathrm{F}(1,176)$ $=.21 ;$ n.s. $)$ et rejettent de manière équivalente $(2.48 / 2.25 ; \mathrm{F}(1,176)=2.21 ;$ n.s. $)$ qu'ils estiment ou non avoir commis des transgressions ou des conduites atypiques en milieu professionnel.

Lorsque les distances vis-à-vis des transgressions de valeurs morales, conventionnelles ou personnelles concernent les proches des sujets, on n'observe ni de différence quant au jugement de gravité $(4.12 / 4.15 ; \mathrm{F}(1,176)=$ .04 ; n.s. $)$ ou au rejet $(3.75 / 3.98 ; \mathrm{F}(1,176)=1.99$; n.s. $)$ des auteurs de conduites transgressives de valeurs morales, ni de différence quant au jugement de gravité $(2.92 / 3.13 ; \mathrm{F}(1,176)=2.99 ;$ n.s. $)$ ou au rejet $(2.75 / 2.99 ; \mathrm{F}(1,176)=$ 2.13 ; n.s.) des auteurs de conduites transgressives de valeurs conventionnelles, ni enfin de différence quant au jugement de gravité $(2.26 / 2.30 ; \mathrm{F}(1,176)=.11$; n.s.) ou au rejet $(2.27 / 2.51 ; \mathrm{F}(1,176)=1.79 ;$ n.s. $)$ des auteurs de conduites transgressives de valeurs personnelles. 
Par contre, lorsque les distances concernent les collègues de travail des sujets, ces derniers tendent à juger plus gravement $(4.10 / 3.31 ; \mathrm{F}(1,176)=3.03$; $\mathrm{p}<.08)$ les conduites transgressives de valeurs morales et rejettent davantage leurs auteurs $(4.07 / 3.74 ; \mathrm{F}(1,176)=4.02 ; \mathrm{p}<.05)$ quant leurs collègues n'ont jamais commis ce type de conduites plutôt que lorsqu'ils en ont commis. De même, ils jugent plus gravement $(3.22 / 2.91 ; \mathrm{F}(1,176)=5.93 ; \mathrm{p}<.01)$ les conduites transgressives de valeurs conventionnelles et tendent à rejeter davantage leurs auteurs $(3.07 / 2.75 ; \mathrm{F}(1,176)=3.58 ; \mathrm{p}<.06)$ quant leurs collègues n'ont jamais commis ce type de conduites plutôt que lorsqu'ils en ont commis. Sur le registre des valeurs personnelles, on n'observe pas de différence concernant les jugements de gravité $(2.25 / 2.42 ; \mathrm{F}(1,176)=1.78 ;$ n.s. $)$. Par contre, comme pour les conduites transgressives, les sujets rejettent davantage les auteurs de conduites transgressives de valeurs personnelles (2.64/2.25; $\mathrm{F}(1,176)=4.58 ; \mathrm{p}<.03)$ quant leurs collègues n'ont jamais exercé ce type de conduites plutôt que lorsqu'ils en ont eu.

3.2 Les relations entre les distances de pouvoir, les distances sociales et les jugements socio-moraux

Les sujets expriment des valeurs de distances de pouvoir plus fortes ( $\mathrm{M}$ $=2.77$ ) lorsqu'ils estiment eux-mêmes ne pas avoir commis de transgressions de valeurs morales, conventionnelles et/ou personnelles en milieu professionnel plutôt que lorsqu'ils estiment en avoir commis $(\mathrm{M}=2.46 ; \mathrm{F}(1,176)=5.30$; $\mathrm{p}<.02)$. De même, ils expriment des valeurs de distances de pouvoir plus fortes $(\mathrm{M}=3.03)$ lorsqu'ils estiment que leurs proches (famille, amis) n'ont jamais commis de transgressions et/ou de conduites atypiques en milieu professionnel plutôt que lorsqu'ils en ont commis $(\mathrm{M}=2.44 ; \mathrm{F}(1,176)=14.89 ; \mathrm{p}<.0002)$. On n'observe pas de différence quant à la perception des transgressions des collègues en relation avec les valeurs de distances de pouvoir (2.51/2.70; $\mathrm{F}(1,176)=1.30 ;$ n.s. $)$.

Par ailleurs, on observe que le jugement de gravité et de rejet est d'autant plus important que les valeurs de distances de pouvoir sont fortes. Précisément, plus les sujets expriment des valeurs de pouvoir fortes et plus ils jugent graves les transgressions de valeurs morales $(r=.27, p<.05)$, de valeurs conventionnelles $(\mathrm{r}=.33, \mathrm{p}<.05)$ et de valeurs personnelles $(\mathrm{r}=.23, \mathrm{p}<.05)$. Dans ce cas, ils rejettent d'autant plus les auteurs de transgressions de valeurs morales $(\mathrm{r}=.38, \mathrm{p}<.05)$, de valeurs conventionnelles $(\mathrm{r}=.31, \mathrm{p}<.05)$ et de valeurs personnelles $(\mathrm{r}=.22, \mathrm{p}<.05)$. 


\section{Discussion}

Les distances vis-à-vis des transgressions ont un impact sur les jugements socio-moraux dès lors qu'ils concernent les conduites des personnes évoluant dans le champ professionnel. Dans ce cas, les personnes évoluant en milieu professionnel jugent plus gravement les actes et rejettent d'autant plus leurs auteurs qu'ils sont eux-mêmes psychologiquement distants de l'acte en estimant ne pas l'avoir commis mais également que des personnes évoluant dans la même sphère professionnelle qu'eux sont eux-mêmes psychologiquement distants de l'acte de transgression. Dans le cas où les transgressions portent sur des valeurs personnelles, ce phénomène s'applique vis-à-vis des collègues mais pas des sujets eux-mêmes ni de leurs proches. La première hypothèse est donc confirmée partiellement, le contexte, renvoyant à la personne en cause (Abdellaoui, Auzoult \& Personnaz, 2010) et au type de valeur en jeu, venant moduler les jugements de sévérité et de rejet.

Par ailleurs, les résultats révèlent que les valeurs de distances de pouvoir sont reliées à la perception des distances psychologiques vis-à-vis des conduites transgressives et/ou atypiques dans la sphère personnelle et intime plutôt que dans la sphère professionnelle des personnes. L'hypothèse 2 est donc vérifiée elle aussi en partie. On observe également une relation entre les valeurs de pouvoir et les jugements de sévérité et de rejet vis-à-vis des transgressions et des conduites atypiques, la sévérité s'accentuant avec la force d'expression des valeurs de pouvoir. L'hypothèse 3 est donc vérifiée.

Globalement, la valeur explicative des distances vis-à-vis des jugements socio-moraux est confirmée. Ces résultats permettent d'établir que le champ psychologique du travail se structure à travers les valeurs des personnes, en l'occurrence ici des valeurs de distances de pouvoir, celles-ci étant reliées aux distances psychologiques que les personnes construisent entre les salariés et leurs conduites éventuelles. Certaines frontières sont perceptibles entre la sphère intime (salariés et proches) dont relèvent les valeurs et la sphère professionnelle à proprement dite (salariés et collègues) où les distances psychologiques déterminent les jugements de sévérité et de rejet. Dans cette perspective plus les distances sont importantes et plus les personnes invoquent les normes pour juger et traiter sévèrement des conduites déviantes mais aussi atypiques dans une certaine mesure. La prise en compte du contexte se traduisant par une plus grande tolérance n'intervenant que lorsque les distances se réduisent vis-à-vis des transgressions.

Ces résultats suggèrent de nouvelles pistes interprétatives concernant l'impact des facteurs organisationnels sur les jugements et les conduites déviantes au travail. Par exemple, on sait que les distances psychologiques 
dépendent des structures organisationnelles (Poppe, 2003 ; Van Dijke \& Poppe, 2003 ; 2007), ces dernières ayant un impact sur les comportements antisociaux au travail (Desrumaux-Zagrodnicki, Lemoine \& Mahon, 2004; Leblanc, LaFrenière, St-Sauveur, Simard, Duval, LeBrock, Girard, Brunet \& Savoie, 2004 ; Flaherty \& Moss, 2007 ; Ménard, Brunet, Savoie, Van Daele \& Flament, 2007). D'autres variables comme le climat, la culture ou la perception de justice organisationnelle pourraient elles aussi être reliées à l'élaboration des distances psychologiques vis-à-vis des conduites au travail.

Cette étude met également en lumière la nécessité de considérer les conduites déviantes au travail en référence à la nature des valeurs qu'elles transgressent. Ce type de distinction permettrait d'analyser plus finement les jugements qui s'élaborent vis-à-vis des conduites déviantes et pourrait permettre d'expliquer la permanence et la tolérance vis-à-vis de certaines conduites dont les salaries sont proches en terme de distance psychologique. Pour illustrer notre propos, nous avons indiqué dans notre méthodologie que la conduite de harcèlement sexuel, qui est pourtant typique des CAAT dans la littérature, n'avait pu être retenue comme un item définitif, celle-ci ayant été évaluée indistinctement comme une transgression et/ou une conduite atypique. Ce résultat suggère pour de futures recherches l'examen des conditions qui conduisent à une relativisation des registres normatifs, ce phénomène amenant potentiellement à traiter toutes les conduites déviantes ou atypiques de manière similaire et indifférenciée.

\section{Références}

Abdellaoui, S., (2000). Stratégies sociocognitives du détenu : recherche d'identité, recherche de contrôle, avant et après jugement. Villeneuve d'Ascq, Presses Universitaires du Septentrion.

Abdellaoui, S., Auzoult, L., Personnaz, B. (2010, Août). Référence à soi, à autrui \& indice de prohibition comparative. Communication pour le 8ème Congrès International de Psychologie Sociale en Langue Française de l'ADRIPS, Nice.

Bandura, A. (1986). Social foundations of Thought and action: A social cognitive theory. Englewood Cliffs, N.J.: Prentice-Hall.

Bègue, L. (1998). De la «cognition morale » à l'étude des stratégies du positionnement moral : aperçu théorique et controverses actuelles en psychologie morale. L'Année Psychologique, 98, 295-352.

Blasi, A. (1988). Bridging moral cognition and moral action: a critical review of the literature, Psychological Bulletin, 1, 1-45.

Blatier, C. (1998). La Délinquance des mineurs : l'enfant, le psychologue, le droit. Grenoble. PUG.

Brockner, J., Ackerman, G., Greenberg, J., Gelfand, M.J., Francesco, A.M., Chen, Z.X., Leung, K., Bierbrauer, G., Gomez, C., Kirkman, B.L., \& Shapiro, D. (2001). Culture and procedural justice: the influence of power distance on reactions to voice. Journal of Experimental Social Psychology, 37, 4, 300-315.

Buss. A. (1979). Self-consciousness and Anxiety. San Francisco : Freeman. 
Caramelli, M. (2006). Une étude des effets de l'actionnariat salarié dans le contexte de l'entreprise multinationale : une approche attitudinale interculturelle. Thèse de Doctorat, Université de Montpellier II.

Carson, J. (2005). Shared Leadership and Culture: Potential Emergence and Global Application. In : Huber, N. S., Walker, M. C. (Eds). Emergent models of global leadership: a volume in building leadership bridges, College Park, MD: The James MacGregor Burns Academy of Leadership, 1-16.

Codol, J. P. (1975). Effet PIP et conflit de normes. L'année psychologique, 75(1), 127145.

Desrumaux-Zagrodnicki, P., Lemoine, C. \& Mahon, P. (2004). Harcèlement moral et climats d'entreprise : effets de facteurs humains et organisationnels sur les jugements d'équité. Psychologie du travail et des organisations, 10, 29-44.

Earley, P. C., \& Erez, M. (1997). The transplanted executive: Why you need to understand how workers in other countries see the world differently. New York : Oxford University Press.

Emler, N. (1987), Socio-moral development from the perspective of social representations, Journal for the Theory of Social Behavior, 17, 371-388.

Eyal, T., Liberman, N., Trope, Y. (2008). Judging near and distant virtue and vice. Journal of Experimental Social Psychology, 44, 1204-1209.

Eylon, D. \& Au, K. Y. (1999). Exploring Empowerment Cross-Cultural differences along the power distance dimension. International Journal of Intercultural Relations, 23, 3, 373-385.

Fischer G.N. (1990). Les domaines de la psychologie sociale. 1 : Le champ du social. Paris : Bordas.

Flaherty, S. \& Moss, S. A. (2007). The impact of personality and team context on the relationship between workplace injustice and counterproductive work behavior. Journal of Applied Social Psychology, 37, 2549-2575.

Gangloff, B. (1995). La valorisation de l'internalité : une hypothèse conditionnelle. Psychologie du Travail et des Organisations, 1, 28-34.

Gangloff, B. (1996). De la norme d'internalité à la norme d'allégeance. Communication du congrès international de psychologie sociale. Montréal.

Gangloff, B. (1997). Les implications théoriques d'un choix d'items : de la norme d'internalité à la norme d'allégeance. Pratiques Psychologiques, 2, 99-106.

Gangloff, B. (2000). Profession recruteur profession imposteur. Paris: L'harmattan.

Gangloff, B. (2002). L'internalité et l'allégeance, considérées comme des normes sociales : Une revue. Les Cahiers de Psychologie Politique. http://www.cahierspsypol.fr.st/

Hofstede, G. (1989). Un réexamen des cultures nationales. Cahiers Internationaux de Psychologie Sociale, 1, 43-64.

Hofstede, G. (2001). Culture's consequences - comparing values, behaviors, institutions and organizations across nations. Thousand Oaks, Sage publications.

House, R. J., Javidan, M., Hanges, P. I., \& Dorfman, P. W. (2002). Understanding Culture and implicit leadership theories across the globe: an introduction to Project Globe. Journal of World Business, 37, 3-10.

Jauvin, N. (2003). La violence organisationnelle : parcours conceptuel et théorique et proposition d'un modèle compréhensif intégrateur, Rapport de recherche, Québec, CLSC CHSLD Haute-Ville-des Rivières.

Kohlberg L. (1958). The development of modes of moral thinking and choice in the years 10-16. Unpublished doctoral dissertation, University of Chicago. 
Kohlberg, L. (1981). Essays on moral development: the philosophy of moral development: moral stages and the idea of justice (vol. 1). San-Francisco : Harper and Row.

Leblanc, G., LaFrenière, A., St-Sauveur, C., Simard, M., Duval, M., LeBrock, P., Girard, C., Brunet, L. \& Savoie, A. (2004). Explication des comportements antisociaux au travail : présentation d'un modèle intégratif. Psychologie du travail et des organisations, 10, 61-73.

Kull, T. J., \& Wacker, J. G. (2009). Quality management effectiveness in Asia: the influence of culture. Journal of Operations Management, doi: 10.1016/j.jom.2009.11.003.

Leblanc, G. LaFrenière, A., St-Sauveur, C., Simard, M., Duval, M., LeBrock, P., Girard, C., Brunet, L. \& Savoie, A. (2004). Explication des comportements antisociaux au travail : présentation d'un modèle intégratif. Psychologie du travail et des organisations, 10, 61-73.

Lee, C., Pillutla, M. \& Law, K.S. (2000). Power-distance, gender and organizational justice. Journal of Management, 26, 4, 685-704.

Masson-Maret, H. \& Steiner, D. D. (2004). Climat de justice et harcèlement moral : perspectives théoriques et outils de diagnostic. Psychologie du travail et des organisations, 10, 9-28.

Ménard, J., Brunet, L., Savoie, A., Van Daele, A. \& Flament, A. (2007). Comportements anti-sociaux au travail : les meilleurs prédicteurs des déviances de production et de propriété. Psychologie du travail et des organisations, 13, 45-62.

Mulder, L.B., Verboon, P. \& De Cremer, D. (2009). Sanctions and moral judgments: the moderating effect of sanction severity and thrust in authorities. European Journal of Social Psychology, 39, 255-269.

Nucci L. et Turiel, E., (1978). Social interactions and the development of social concepts in presschool children. Child development, 49, 400-407.

Nucci, L. (1981). Conceptions of personal concept : A domain distinct from moral or societal concepts. Child development, 52, 114-121.

Piaget, J. (1932). Le jugement moral chez l'enfant. Paris : PUF.

Poppe, M. (2003). Preferences for changing power positions and power distances : a social value orientations approach. European Journal of Social Psychology, 33, 2, 177-193.

Roché, S. (2001). La délinquance des jeunes : Les 13-19 ans racontent leurs délits. Paris : Seuil.

Rom, N. R. A, HI Hsu, C. (2002). Reconsidering the exploration of power distance: an active case study approach. Omega, 30, 403-414.

Shweder, R. A. (1982). On savages and other children. American anthropologist, 84, 354-366.

Tan, W., Chon, E. (2003). Power distance in Singapore construction organization: implications for project managers. International Journal of Project Management, 21, 529-536.

Tap P. (1988). La société pygmalion? Intégration sociale et réalisation de la personne. Paris : Dunod.

Tostain, M. (1999a). Psychologie, morale et culture. L'évolution de la morale de l'enfance à l'âge adulte. Grenoble : Presses Universitaires de Grenoble.

Tostain, M. (1999b). La morale est-elle universelle ? In J.-L. Beauvois, N. Dubois, W. Doise (Eds.). La construction sociale de la personne (pp. 47-58). Grenoble: P.U.G. 
Turiel, E., 1983. The development of social knowledge: Morality and convention. Cambridge, England : Cambridge University Press.

Van de Vliert, E., \& Smith, P.B. (2004). Leader reliance on subordinates across nations that differ in development and climate. The Leadership Quarterly, 15, 381-403.

Vandenplas-Holper, C. (1999). Piaget, Kohlberg et les «postkohlbergiens »: plus d'un demi-siècle de recherches concernant le developpement moral. In J.-L. Beauvois, N. Dubois, W. Doise (Eds.). La construction sociale de la personne (pp. 21-46). Grenoble: P.U.G.

Van Dijke, M., \& Poppe, M. (2003). Preferred changes in power differences: effects of social comparison in equal and unequal power relations. Journal of Experimental Social Psychology, 39, 149-160.

Van Dijke, M. \& Poppe, M. (2007). Motivations underling power dynamics in hierarchically structured groups. Small Group Research, 38, 6, 643-669. 
Annexe 1 : Conduites transgressives et atypiques en milieu professionnel

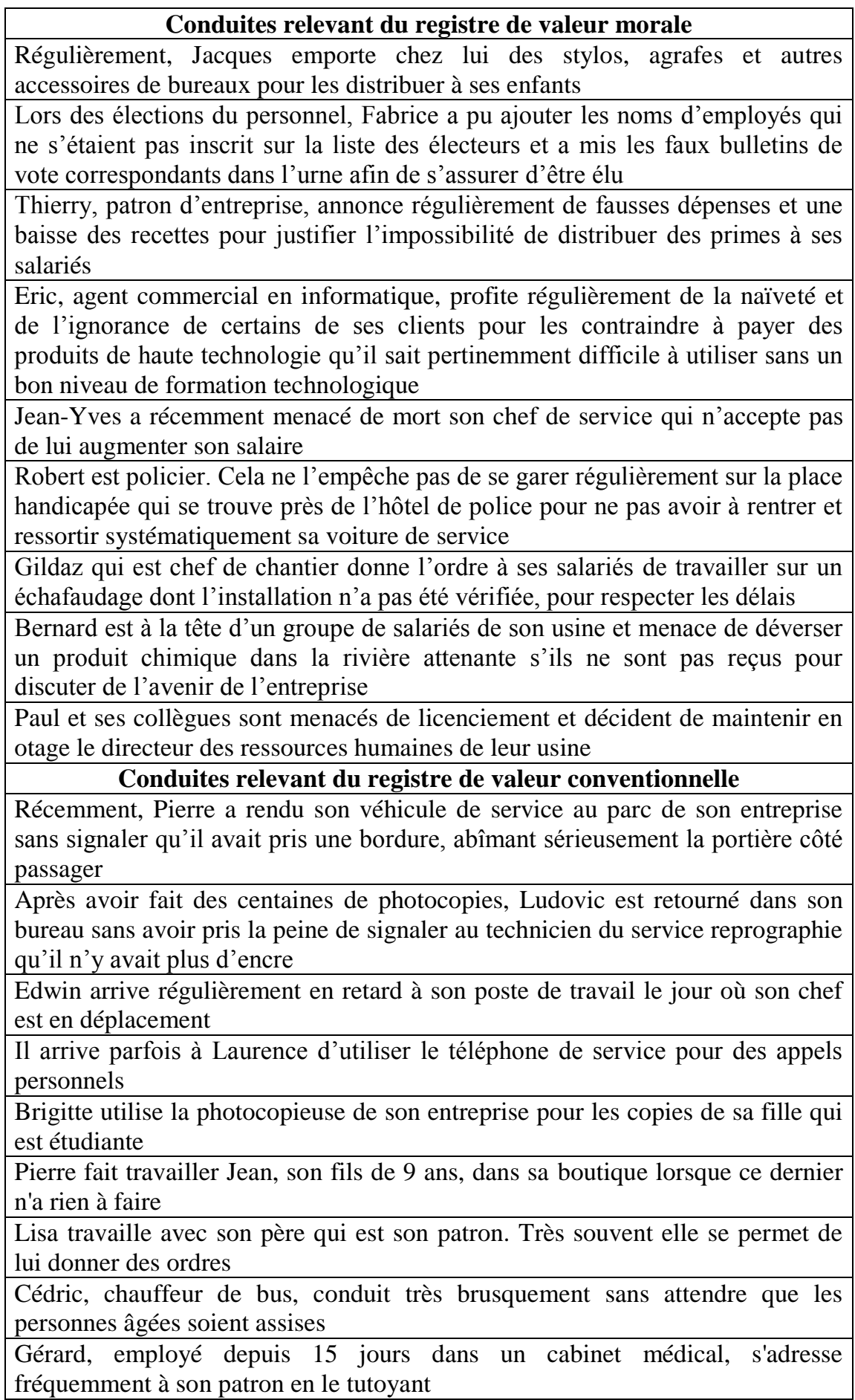




\begin{tabular}{|l|}
\hline \multicolumn{1}{|c|}{ Conduites relevant du registre de valeur personnelle } \\
\hline $\begin{array}{l}\text { Tous les lundis, Roger, chef magasinier dans une société de conditionnement de } \\
\text { fruits et légumes, se rend à son travail en jogging rose }\end{array}$ \\
\hline $\begin{array}{l}\text { Il arrive souvent à Dominique de s'asseoir en tailleur sur sa chaise de bureau } \\
\text { pour travailler }\end{array}$ \\
\hline $\begin{array}{l}\text { Mettre des chaussures de sécurité n'est pas une priorité pour Bruno, ouvrier } \\
\text { dans le bâtiment. Il lui arrive même régulièrement de travailler en espadrille }\end{array}$ \\
\hline $\begin{array}{l}\text { Noël prend du plaisir à collectionner les post-it usagés par ses collègues et par } \\
\text { lui-même }\end{array}$ \\
\hline $\begin{array}{l}\text { Il arrive à Marc de venir en short au travail malgré la demande de sa hiérarchie } \\
\text { de porter un uniforme }\end{array}$ \\
\hline Patrick visite des sites de rencontre sur Internet le midi pendant sa pause \\
\hline Jean Marie, juge d'instruction est coiffé d'une crête punk \\
\hline Au travail, Timothée se déguise en fonction de chaque fête du calendrier \\
\hline Jean commercial reçoit ses clients en baskets \\
\hline
\end{tabular}

